\title{
What we still don't know about treating chronic noncancer pain with opioids
}

\author{
Roger Chou MD \\ Previously published at www.cmaj.ca
}

I n a survey of 2012 Canadian adults, nearly one-third reported chronic noncancer pain, and the average duration of pain was over 10 years. ${ }^{1}$ Other data show that opioids are commonly — and increasingly - used to treat chronic noncancer pain. ${ }^{2}$ At the same time, many clinicians report discomfort, uncertainty or frustration about opioid treatment, indicating a need for additional guidance. To address these concerns, a new Canadian practice guideline for the use of opioids to treat chronic noncancer pain has been developed. It is summarized in this issue by Furlan and colleagues. ${ }^{3}$

Although other Canadian guidelines on the use of opioids in treating chronic noncancer pain have been issued previously, ${ }^{4,5}$ what helps distinguish the new guideline is the rigorous process used to develop it. The new guideline is the product of a large, multidisciplinary group. Stakeholders were extensively involved, a systematic review of evidence was conducted, and the recommendations were clearly linked to the supporting evidence. The guideline also includes tools to help clinicians implement the recommendations in practice. These methods generally follow published standards for developing guidelines, which should reassure potential users. ${ }^{6}$

The Canadian effort follows (by about one year) an American guideline ${ }^{7}$ sponsored by the American Pain Society and American Academy of Pain Medicine. The recommendations in the two guidelines are quite consistent. Both recommend a judicious approach to use of opioids, with an emphasis on risk stratification, monitoring to determine appropriateness of continued therapy and to mitigate potential harms, and the need to appropriately address aberrant drug-related behaviours (which in some cases could mean discontinuation of opioid therapy).

Unfortunately, another aspect of the two guidelines is similar. In the Canadian guideline, just 3 of 24 recommendations were classified as based on randomized controlled trials. Nineteen recommendations were based solely or partially on consensus opinion. In the United States guideline, 21 of 25 recommendations were viewed as supported by only lowquality evidence. In other words, the developers of the guidelines found that what we know about opioids is dwarfed by what we don't know.

The continued failure of research to address clinically relevant issues related to prescribing of opioids is disappointing, but is not cause to dismiss the guidelines out of hand. The

\section{Key points}

- Because of a lack of strong evidence, most recommendations in the new Canadian guideline for the use of opioids to treat chonic noncancer pain are primarily based on consensus opinion.

- The best evidence supporting opioid use for chronic noncancer pain comes from short-term trials of efficacy in highly selected populations.

- Research into the effectiveness and comparative effectiveness of opioid use in higher-risk populations with long-term follow-up is needed, along with studies to address other research gaps.

- Until such studies are completed, the new Canadian guideline provides clinically sound recommendations for decision-making.

process of reviewing evidence and generating recommendations is what makes a guideline evidence-based. ${ }^{6}$ Sometimes the evidence is strong - in which case generating recommendations is usually straightforward - and sometimes it isn't. When it isn't, thoughtful recommendations can still provide the best current guidance, given that the evidence is thoroughly considered, potential conflicts of interest are appropriately managed, clinical reasoning is sound, and research gaps are clearly defined. The delineation of research gaps, an overlooked aspect of the process of guideline development, can in itself be of high value.

What are the critical gaps in research associated with use of opioids for chronic noncancer pain, and how might these gaps be addressed? It might be easier to start by summarizing what we do know with some certainty. A meta-analysis of randomized, placebo-controlled trials that were primarily short-term (i.e., typically less than eight weeks) showed that treatment with opioids is associated with moderate improvements in pain (less so for improvements in function) in highly selected, low-risk populations with chronic noncancer pain. ${ }^{8}$ Such efficacy-based evidence can be useful for certain purposes (e.g., for gaining regulatory approval), but often doesn't

Roger Chou is with the Departments of Medicine and Medical Informatics \& Clinical Epidemiology, Oregon Health and Science University, Portland, USA

CMAJ 2010. DOI:10.1503/cmaj.100548

All editorial matter in CMAJ represents the opinions of the authors and not necessarily those of the Canadian Medical Association. 
translate well into clinical practice. Clearly, we need longerterm studies that evaluate the higher-risk patients commonly encountered by clinicians.

We need to know a lot more than simply whether an opioid works or not. Appropriate use of opioids involves many decisions. We need research to develop and validate practical instruments for prediction of risk, and ultimately to understand how using them to guide treatment decisions affects patient outcomes. ${ }^{9}$ We also have very limited data on the comparative benefits and harms of different opioids (particularly with regard to rates of addiction or abuse) or different methods of prescribing opioids (e.g., around-the-clock, longacting opioids versus as-needed or short-acting opioids). ${ }^{10}$ Methadone, for example, differs from other opioids because it has a long and variable half-life and is associated with prolongation of the electrocardiographic QT interval. Epidemiologic data indicate large increases in methadone-associated fatalities, ${ }^{11}$ yet almost no evidence exists on risks of methadone versus other opioids, or on the effectiveness of strategies to mitigate potential risks.

We also don't know the most appropriate methods for monitoring patients. For example, we don't have evidence to guide how frequently to perform urinary screening for drugs, what components to include in the screening, or how patient outcomes are affected by urinary drug screening. ${ }^{9}$ We have little evidence on optimal methods for discontinuing opioids, and don't know the long-term outcomes in patients taken off therapy. We also have little research to guide use of higher doses of opioids. A recent, well-conducted observational study found that higher doses are associated with increased mortality risk, suggesting that recommendations for cautious use of high doses are warranted. ${ }^{12}$ However, no studies have compared the benefits and harms of further dose escalations versus alternative treatment strategies (e.g., opioid rotation, replacement with an alternative medication, dose reduction, or addition of nonpharmacological therapy) in patients taking higher doses.

The principles of research into comparative effectiveness are well suited for addressing these and other research gaps. ${ }^{13}$ Rather than evaluating whether yet another opioid is more effective than nothing in low-risk patients, such research focuses on the benefits and harms of interventions in populations similar to those encountered in clinical practice, emphasizing the need to understand the trade-offs between different interventions (e.g., different opioids). These principles can be applied to the evaluation of different strategies for risk assessment, patient selection, dosing, management and monitoring, using a broad range of study designs, including observational studies of large databases or registries and randomized trials that examine effectiveness. For researchers, many funding opportunities - and challenges - related to research into comparative effectiveness are currently available.

Research into comparative effectiveness is likely to raise as many questions about opioids as it answers. Nonetheless, we should expect new research to challenge our beliefs about how to prescribe these medications. Recommendations based on weak evidence are associated with a low degree of certainty and subject to change. Indeed, we should be glad if new research causes us to rethink current practices; it would be a sign that we finally understand enough to move forward knowledgeably. While waiting for the research that is still needed, clinicians can use the new Canadian guideline as a clinically sensible framework for decisions that need to be made now.

Competing interests: Roger Chou is director of Guideline Development at the American Pain Society.

\section{REFERENCES}

1. Moulin DE, Clark AJ, Speechley M, et al. Chronic pain in Canada - prevalence, treatment, impact and the role of opioid analgesia. Pain Res Manag 2002;7:179-84

2. Boudreau D, Von Korff M, Rutter CM, et al. Trends in long-term opioid therapy for chronic non-cancer pain. Pharmacoepidemiol Drug Saf 2009;18:1166-75.

3. Furlan AD, Reardon R, Weppler C. Opioids for chronic noncancer pain: a new Canadian practice guideline. CMAJ 2010;182:923-30.

4. Hagen N, Flynne P, Hays H, et al. Guidelines for managing chronic non-malignant pain. Opioids and other agents. Can Fam Physician 1995;41:49-53.

5. Jovey RD, Ennis J, Gardner-Nix J, et al. Use of opioid analgesics for the treatment of chronic noncancer pain - a consensus statement and guidelines from the Canadian Pain Society, 2002. Pain Res Manag 2003;8(Suppl. A):3A-28A.

6. Appraisal of guidelines for research \& evaluation. The AGREE Collaboration; 2001. Available: www.agreecollaboration.org/pdf/agreeinstrumentfinal.pdf (accessed 2010 Apr. 14)

7. Chou R, Fanciullo G, Fine P, et al. Clinical guidelines for the use of chronic opioid therapy in chronic noncancer pain. J Pain 2009;10:113-30

8. Furlan AD, Sandoval JA, Mailis-Gagnon A, et al. Opioids for chronic noncancer pain: a meta-analysis of effectiveness and side effects. CMAJ 2006;174:1589-94.

9. Chou R, Fanciullo G, Fine PG, et al. Opioids for chronic noncancer pain: prediction and identification of aberrant drug-related behaviors. A review of the evidence for an American Pain Society and American Academy of Pain Medicine clinical practice guideline. J Pain 2009;10:131-46.

10. Chou R, Ballantyne JC, Fanciullo GJ, et al. Research gaps on use of opioids for chornic noncancer pain: findings from a review fo the evidence for an American Pain Society and American Academy of Pain Medicine Clinical Practice Guideline. J Pain 2009;10:147-59.

11. Methadone-associated mortality: report of a national assessment, May8-9, 2003. Washington (DC): Center for Substance Abuse Treatment; 2004. Available: http://pain-topics.org/pdf/CSAT_Methadone_Briefing.pdf (accessed 2010 Apr. 14).

12. Dunn KM, Saunders KW, Rutter CM, et al. Opioid prescriptions for chronic pain and overdose. A cohort study. Ann Intern Med 2010;152:85-92.

13. Sox HC, Greenfield S. Comparative effectiveness research: a report from the Institute of Medicine. Ann Intern Med 2009;151:203-5.

Correspondence to: Dr. Roger Chou, Oregon Health and Science University, 3181 SW Sam Jackson Park Rd., Portland OR 97239. 3098,USA; chour@ohsu.edu 